\title{
Estudiantes de sectores vulnerables con alto rendimiento escolar y su desempeño en variables intelectuales, resiliencia, autoestima y bienestar psicológico ${ }^{1}$
}

Senior high school students of vulnerable schools who have high performances and results in variables intelectual, resilence, self esteem and psychological well-being

\author{
Mario Morales ${ }^{2}$ \\ Universidad de Santiago de Chile, Santiago, Chile \\ (RECIBIDO 04/01/2014, ACEPTADO 24/03/2014)
}

\begin{abstract}
RESUMEN
El artículo sintetiza los resultados de una investigación en la cual se evaluaron a estudiantes de cuarto año de enseñanza media con alto rendimiento escolar, pertenecientes a sectores vulnerables. El objetivo central del estudio fue comparar este grupo de alumnos con el grupo total, en variables relacionadas con la capacidad intelectual, autoestima, resiliencia y bienestar psicológico. Se recabó la información a través de distintas escalas, todas ellas arrojaron niveles de confiabilidad sobre 0.70 (alfa de Cronbach). Los resultados revelaron que los rendimientos en las diversas escalas se encuentran dentro del rango esperado para su edad. Sin embargo, las diferencias entre los grupos de rendimiento alto y rendimiento general solo se evidencian en el rendimiento intelectual, escala de autoestima (hogar), resiliencia (modelos y aprendizaje) y bienestar psicológico (aceptación y proyección).
\end{abstract}

Palabras clave: alto rendimiento, vulnerabilidad, factores psicosociales, capacidad intelectual

\begin{abstract}
The article summarizes the results of an investigation assessing a group of students of senior high from vulnerable schools. The central objective of the study was to compare their averages on the various scales with those students who rank average or below in the class groups in variables related to the student's intellectual capacity, self-esteem, resilience and psychological well-being. Data was collected through different scales, all of them showed levels of reliability above 0.70 (Cronbach Alpha).
\end{abstract}

1 Investigación financiada por Departamento de Investigaciones Científicas y Tecnológicas DICYT. Universidad de Santiago de Chile

2 Docente de la Escuela de Psicología de la Universidad de Santiago de Chile. Email: mario.morales@usach.cl 
ESTUDIANTES DE SECTORES VULNERABLES CON ALTO RENDIMIENTO ESCOLAR Y SU DESEMPEÑO EN VARIABLES INTELECTUALES...

The results indicated that performance is within the expected range for their age. However, the significant differences between the groups of high performance and the rest of the group were only evident in the intellectual performance; Self esteem (scale home); resilience (learning y models) and psychological well-being (acceptance and projection).

Keywords: Higher achievers, vulnerability, psychosocial factors, intellectual capacity.

\section{INTRODUCCIÓN}

Una de las preocupaciones de las políticas educativas de los diversos países de Latinoamérica es resolver el problema de inequidad y desigualdad en el sistema educativo. Entre las escuelas que provienen de sectores más acomodados y aquellas que provienen de sectores social y económicamente más vulnerables hay una brecha importante.

Las condiciones asociadas a las escuelas de los sectores más desfavorecidos exponen a los estudiantes a numerosos factores de riesgo que comprometen su calidad de vida y funcionamiento psicológico. Sin embargo, el daño potencial ocasionado por todas las situaciones que se vinculan con la pobreza no es inevitable ni irreversible. Existen factores protectores que amortiguan el impacto de las diferentes clases de privación a las que están expuestos estos alumnos, como son las emociones positivas que pueden contrarrestar e incluso prevenir los efectos nocivos que acarrean las vivencias derivadas de la pobreza (Seligman, 2005).

La condición de vulnerabilidad afecta el rendimiento escolar por diversos factores; contextuales, salud mental de los padres, la estimulación sensorial y del lenguaje y las características del ambiente físico. Sameroff et al. (1998) mencionan que la exposición a una mayor cantidad de factores de riesgo en la infancia debilita el desarrollo socioemocional y aumenta la probabilidad de desajuste psicológico. Elder et al. (1985) señalan que estos problemas no surgen como resultados directos de las condiciones de pobreza crónica, sino que esta ejerce un efecto indirecto en el escolar mediante su impacto en el estado emocional de los padres. Pese a ello, no todos los estudiantes manifiestan problemas afectivos. Con un adecuado funcionamiento emocional, estos han podido mantener un balance entre los factores de riesgo y los factores protectores, lo que les ha permitido resistir el impacto de los estresores (Werner, 1990). Es así como resultados de investigaciones señalan que estudiantes en algunos sectores más desfavorecidos alcanzan buenos resultados académicos (Téllez, 2005; Rodríguez y Valdivieso, 2008).

Una mirada desde la psicología positiva, cuyo objetivo es identificar los determinantes individuales y colectivos de las experiencias positivas para entender las causas y las consecuencias del bienestar psicológico en sí mismo (Seligman, 2005), nos permitiría comprender distintas características individuales que poseen aquellos alumnos que logran alto rendimiento escolar a pesar de diversos factores ambientales, familiares y escolares adversos que caracterizan a estas instituciones.

El objetivo de la presente investigación fue analizar las variables que influyen en el alto rendimiento que presentaban un grupo de estudiantes de cuarto año 
de enseñanza media provenientes de liceos prioritarios. La pregunta central de investigación fue: ¿Qué diferencias existen entre aquellos estudiantes de altos rendimientos y los demás compañeros de la misma clase en factores intelectuales, resiliencia, autoestima y bienestar psicológico?

La hipótesis que sustenta este estudio es que existen diferencias significativas entre los grupos de estudiantes de alto rendimiento y el grupo general en variables relacionadas con la capacidad intelectual, resiliencia, autoestima y bienestar psicológico.

La estrecha vinculación entre inteligencia y capacidad de aprendizaje es evidente, sin embargo las cuestiones relativas a la relación entre inteligencia, aptitudes para el aprendizaje y rendimiento académico distan mucho de estar realmente resueltas. La inteligencia y las aptitudes son las variables que con mayor frecuencia son consideradas predictoras del rendimiento académico, sin embargo, en la mayoría de los estudios, los factores exclusivamente intelectuales explican, en el mejor de los casos, alrededor de un $25 \%$ de la varianza del rendimiento académico (Aliaga, 1998). Por tanto, si bien la inteligencia general explica una parte importante del rendimiento escolar de los alumnos, es necesario examinar otras variables que sean capaces de determinar el resto de la varianza.

Los factores personales asociados al rendimiento escolar que fueron analizados son: autoestima, resiliencia y bienestar personal. El primer factor, la autoestima, comprende dos aspectos complementarios: la íntima convicción de tener valor como persona y la percepción de la capacidad personal (Polleti y Dobbs, 2005). Existe consenso entre los autores para entender la autoestima como la capacidad de conocerse a sí mismo, de velar por uno, autodefenderse, valorarse, estimarse y autoobservarse (Acosta y Hernández, 2004). Por otra parte, ha sido definida como un proceso dinámico y multidimensional que se construye a lo largo de toda la vida mediante la exposición a diferentes contextos, ya sea cognitivo, emocional, social y físico (Naranjo, 2007). Este sentimiento valorativo configura la personalidad, siendo un producto social resultante de la interacción del sujeto con su entorno y se encarga de dirigir la actividad del cuerpo y la mente de las personas (Acosta y Hernández, 2004). Así también, facilita la incorporación de la responsabilidad para dirigir la vida, en conjunto con el autoconocimiento y autocontrol, lo que permite mantener armonía y una buena convivencia con el ambiente, así como habilidades cognitivas para progresar en la formación académica (González-Pineda et al., 2003). Conjuntamente, como plantea Haeussler y Milicic (1995), los sujetos que se caracterizan por tener una buena autoestima tienen alta motivación intrínseca que les facilita el aprendizaje, generan mayores expectativas sobre sí mismos y tienen mejor rendimiento académico que aquellos que no la poseen.

Otra variable que hemos asociado al rendimiento escolar es la resiliencia. Esta ha sido entendida como la capacidad del individuo de hacer las cosas bien, pese a las circunstancias desfavorables; implica una capacidad de resistencia y una facultad de construcción positiva. Masten (2001) alude a la capacidad del ser humano para hacer frente a las adversidades de la vida, superarlas y salir transformado por ellas. 
Grotberg (2006) hace referencia a una adaptación positiva frente a los riesgos o adversidades. El término se relaciona con la existencia de factores de riesgo y factores de protección. Los primeros se construyen en la interacción social, donde se combinan factores internos, como el temperamento, la autoestima, el sentido del humor, entre otros; como del entorno: relaciones afectivas, estímulos, influencia del contexto, entre otros (Kotliarenco et al., 1996).

Existe concordancia entre los autores en que la resiliencia no se puede reducir a un conjunto de rasgos personales, sino que contemplaría factores que operan dentro del individuo y que son cultivados a lo largo de su historia personal (Villalta, 2009). Esto supone el desarrollo de conductas de competencia social, académica y vocacional. Finn y Rock (1997) hablan de resiliencia educacional para referirse a alumnos pertenecientes a minorías raciales de escasos recursos que presentan notas a un nivel aceptable y logran completar la educación escolar. Estos alumnos se caracterizan por manifestar conductas de compromiso con el trabajo escolar, tales como asistencia a clases, puntualidad, poner atención al profesor, realizar las tareas escolares; por altos niveles de autoconfianza, autodisciplina, actitud positiva hacia el trabajo, locus de control interno y autoestima positiva, rasgos que favorecen el aprendizaje.

La expresión resiliencia describe el proceso en el que convergen factores personales y ambientales en la resistencia relativa a experiencias de riesgo psicosocial (Rutter, 1999; Masten, 1994; Torre de Carvalho et al., 2007).

Nuestro tercer factor, el bienestar subjetivo, se relaciona con la subjetividad personal (Diener et al., 1999). Es un concepto cuyas definiciones se podrían caracterizar a partir de tres elementos: su carácter subjetivo, que descansa sobre la propia experiencia de la persona; su dimensión global, porque incluye la valoración del sujeto en todas las áreas de su vida; y la apreciación positiva, ya que su naturaleza va más allá de la mera ausencia de factores negativos (Diener, 1994). La revisión de los estudios sobre el bienestar subjetivo señala que existe acuerdo entre los distintos investigadores con respecto a los elementos que componen su estructura. Se pueden diferenciar dos grandes dimensiones: la dimensión cognitiva y la dimensión emocional o afectiva (Diener et al., 1999).

Podríamos decir, entones, que es el resultado de la valoración global mediante la cual, a través de la articulación de los aspectos afectivos y cognitivos, el sujeto tiene en cuenta tanto su estado anímico presente, como la congruencia entre sus logros alcanzados y sus expectativas sobre una serie de dominios vitales, así como en conjunto sobre la satisfacción con su vida (García Martín, 2004). En este sentido, lo subjetivo no se opone a lo objetivo sino que se complementan. Por otra parte, el bienestar psicológico depende de la congruencia entre las aspiraciones de una persona, la percepción que tiene de sí mismo y de su entorno y la posibilidad de cambiar o realizar acciones positivas en sus circunstancias de vida.

En la adolescencia se ha observado que la autoestima es uno de los predictores de mayor fuerza y consistencia del bienestar psicológico, además estudios han 
mostrado que las percepciones de los adolescentes sobre las relaciones familiares son de mayor importancia para el bienestar que las experiencias escolares entre iguales o las condiciones demográficas (Diener y Lucas 2000; Dew y Scott, 2002; Barber y Weichold, 2006).

Desde otra perspectiva, las condiciones adversas no siempre tienen el mismo efecto negativo en los escolares. El alto rendimiento escolar que presentan los estudiantes actúa como factor protector frente a las adversidades, lo que explicaría, desde la perspectiva de la psicología positiva, que las emociones positivas pueden optimizar la salud, el bienestar subjetivo y la resiliencia psicológica (Fredrickson y Joiner, 2002). Las emociones positivas favorecen un razonamiento eficiente, flexible y creativo. Un razonamiento de este tipo es predictor de un aprendizaje significativo y, por tanto, de un buen rendimiento académico. Mientras que la ansiedad, la depresión y las experiencias de fracaso disminuyen la atención, las emociones positivas enriquecen las funciones cognitivas y mejoran la focalización atencional (Fredrickson y Joiner, 2002).

\section{MÉTODO}

Se trata de un estudio cuantitativo en el que se evaluaron a estudiantes de secundaria (cuarto año de enseñanza media). Se seleccionaron tres establecimientos pertenecientes a liceos prioritarios. Estos establecimientos poseen cerca de un $85 \%$ de índice de vulnerabilidad. Se evaluaron 393 estudiantes. El promedio de edad era de 18.4 años, con una desviación estándar de 1.5 . El $40.6 \%$ eran varones y un 59.4\% mujeres. Del grupo total, se seleccionaron 73 estudiantes que presentaban un rendimiento escolar alto, definido como una desviación estándar sobre el promedio de notas de los últimos tres años.

Se administraron los siguientes instrumentos:

Test de matrices progresivas (Raven, 1991). Evalúa la capacidad del sujeto para comprender los principios que subyacen en un problema. Es una prueba no verbal que evalúa el factor " $\mathrm{g}$ " de la inteligencia. El sujeto debe deducir relaciones entre algunas figuras geométricas y luego encontrar el correlato adecuado. Estas capacidades están relacionadas con la habilidad deductiva, definida como la habilidad para extraer correlatos de informaciones desorganizadas, las cuales no son evidentes al observador de forma inmediata (Ivanovic et al., 2000). El nivel de confiabilidad del instrumento es de 0.78 (alfa de Cronbach).

Inventario de autoestima de Coopersmith (Brinkmann et al., 1989). Es una prueba individual y colectiva. Está dividida en los siguientes subtest: subtest de sí mismo (general), en el cual los puntajes altos indican valoración de sí mismo y altos niveles de aspiración, estabilidad, confianza, adecuadas habilidades sociales y atributos personales; subtest social pares, una puntuación alta indica que el sujeto posee mayores competencias y habilidades en las relaciones con amigos y colaboradores, así como con extraños; subtest hogar 
padres, un nivel alto revela adecuadas cualidades y habilidades en las relaciones íntimas con la familia, el sujeto se siente respetado, tiene independencia y una concepción moral propia; y subtest escolar, los niveles altos indican que el individuo afronta adecuadamente las principales tareas académicas y son personas que poseen buena capacidad para aprender, trabajan la satisfacción tanto a nivel individual como grupal. El nivel de confiabilidad es de 0.70 (alfa de Cronbach).

Escala de resiliencia. La escala de resiliencia SV-RES para jóvenes y adultos (Saavedra y Villalta, 2008) se basa teóricamente en los modelos sobre el constructo de resiliencia de los autores Grotberg (1996) y Saavedra (2003). Fue validada en el año 2007, probándose en población urbana chilena entre 15 y 65 años. Es un instrumento que puede ser aplicado individual y colectivamente e incluso puede ser autoadministrado. Cuenta con 60 ítems con 5 alternativas en escala likert, que van desde "muy de acuerdo" a "muy en desacuerdo". Permite obtener un puntaje global de resiliencia y un puntaje en las siguientes áreas: identidad, autonomía, satisfacción, pragmatismo, vínculos, redes, modelos, metas, afectividad, autoeficacia, aprendizaje y generatividad. El nivel de confiabilidad de la escala es de 0.74 (alfa de Cronbach).

Escala de bienestar psicológico (Bieps). Elaborada por Casullo (2000), consta de 13 reactivos, cuyas puntuaciones van de 1 (en desacuerdo) hasta 3 (de acuerdo). Las dimensiones que evalúa son: aceptación/control, vínculos, proyectos de vida, autonomía. El instrumento posee un nivel de confiabilidad y validez adecuado (0.76). Se ha utilizado en otras investigaciones relacionadas con estudios de adolescentes.

\section{RESULTADOS}

Se procedió a evaluar la normalidad de todas las variables con la prueba de Kolmogorov-Smirnov (K-S), para decidir el uso de prueba t de Students o la U. de Mann-Whitney. Los resultados indicaron que todas las variables medidas no presentaban una distribución normal, por lo que se decidió utilizar una prueba no paramétrica.

Tabla 1. Comparación del grupo de rendimiento alto (RA) con rendimiento general (RG) en el test de matrices progresivas. Raven.

\begin{tabular}{llll}
\hline Categoría & Media & D.S. & Rango medio \\
\hline Rendimiento alto (RA) $(\mathrm{N}=73)$ & 45.62 & 4.54 & 262.11 \\
Rendimiento general $(\mathrm{RG})(\mathrm{N}=320)$ & 41.41 & 6.11 & 182.15 \\
\hline
\end{tabular}

Se puede observar en la tabla 1 que los estudiantes de rendimiento alto obtienen un mejor puntaje en la escala Raven que los alumnos del grupo general. Esta diferencia de acuerdo a la prueba $U$ de Mann-Whitney fue significativa $Z=-5.43(p=.000)$. 
Se aprecia que existen mejores habilidades de los estudiantes de rendimiento alto en habilidad para comparar formas y razonar por analogía, con independencia de los conocimientos adquiridos.

Tabla 2. Comparación del grupo de rendimiento alto con rendimiento general en el inventario de autoestima (Coopersmith)

\begin{tabular}{|c|c|c|c|c|c|}
\hline & Puntaje total & General* & Social & Hogar* & Escolar \\
\hline $\begin{array}{c}\text { Rendimiento alto (RA) } \\
\begin{array}{c}(\mathrm{n}=73) \text { Media } \\
\text { D.S. } \\
\text { Rango medio }\end{array}\end{array}$ & $\begin{array}{c}55.59 \\
9.71 \\
242.6\end{array}$ & $\begin{array}{c}51.78 \\
8.87 \\
204.43\end{array}$ & $\begin{array}{c}50.68 \\
12.88 \\
178.56\end{array}$ & $\begin{array}{c}52.15 \\
11.81 \\
221.53\end{array}$ & $\begin{array}{c}54.47 \\
10.36 \\
210.75\end{array}$ \\
\hline $\begin{array}{l}\text { Rendimiento general (RG) } \\
\qquad \begin{array}{c}(\mathrm{n}=320) \text { Media } \\
\text { D.S. } \\
\text { Rango medio }\end{array}\end{array}$ & $\begin{array}{c}51.09 \\
10.46 \\
186.60\end{array}$ & $\begin{array}{c}50.83 \\
10.21 \\
195.30\end{array}$ & $\begin{array}{c}53.89 \\
10.83 \\
201.21\end{array}$ & $\begin{array}{c}49.33 \\
10.17 \\
191.40\end{array}$ & $\begin{array}{c}52.79 \\
11.17 \\
193.86\end{array}$ \\
\hline & $\begin{array}{l}\mathrm{Z}=-3.80 \\
(\mathrm{p}=.000)\end{array}$ & & & $\begin{array}{l}\mathrm{Z}=-2.05 \\
(\mathrm{p}=.040)\end{array}$ & \\
\hline
\end{tabular}

Se puede apreciar en la tabla 2 que, en el puntaje total de la escala, la diferencia entre ambos grupos aparece significativa. La prueba U de Mann-Whitney arroja un $\mathrm{Z}=-3.80(\mathrm{p}=.000)$. Por otra parte, en las subescalas general, social y escolar no se evidencian diferencias significativas. En la subescala hogar, se observan diferencias significativas: los estudiantes de rendimiento alto obtienen una mayor puntuación en comparación con el grupo general en el nivel de aceptación, con el que la persona valora su conducta en relación con su contexto familiar. Esta diferencia, de acuerdo a la prueba $U$ de Mann-Whitney, fue significativa: $\mathrm{Z}=$ $-2.05(\mathrm{p}=.040)$.

Tabla 3. Comparación del grupo de rendimiento alto (RA) con rendimiento general $(\mathrm{RG})$ en la escala de resiliencia

\begin{tabular}{|c|c|c|c|c|c|c|}
\hline Grupos & & Identidad & Autonomía & \multicolumn{2}{|c|}{ Satisfacción } & \multirow{2}{*}{$\begin{array}{c}\text { Pragmatismo } \\
55.02\end{array}$} \\
\hline \multirow{3}{*}{$\begin{array}{l}\text { R. A. } \\
(73)\end{array}$} & Media & 59.58 & 61.08 & \multicolumn{2}{|c|}{49.54} & \\
\hline & D.S. & 27.68 & 27.43 & \multicolumn{2}{|c|}{27.31} & 27.70 \\
\hline & R. Medio & 193.81 & 204.01 & \multicolumn{2}{|c|}{187.37} & 200.34 \\
\hline \multirow{3}{*}{$\begin{array}{l}\text { R.G. } \\
(320)\end{array}$} & Media & 60.15 & \multirow{3}{*}{$\begin{array}{c}58.76 \\
28.31 \\
195.40\end{array}$} & \multirow{3}{*}{\multicolumn{2}{|c|}{$\begin{array}{c}52.54 \\
26.55 \\
199.20\end{array}$}} & 54.34 \\
\hline & D.S. & 27.34 & & & & 27.57 \\
\hline & R. Medio & 197.73 & & & & 196.24 \\
\hline Grupos & & Vínculos & Redes & Modelos & Metas & Afectividad \\
\hline \multirow{3}{*}{$\begin{array}{l}\text { R. A. } \\
(73)\end{array}$} & Media & 52.71 & 57.31 & 66.95 & 56.03 & 54.51 \\
\hline & D.S. & 29.39 & 30.06 & 15.77 & 25.31 & 28.61 \\
\hline & R.Medio & 196.47 & 199.18 & 230.72 & 200.28 & 196.58 \\
\hline
\end{tabular}


ESTUDIANTES DE SECTORES VULNERABLES CON ALTO RENDIMIENTO ESCOLAR Y SU DESEMPEÑO EN VARIABLES INTELECTUALES...

\begin{tabular}{ccccccc} 
R.G. & Media & 53.09 & 56.78 & 56.88 & 62.25 & 55.61 \\
$(320)$ & D.S. & 26.71 & 28.76 & 28.97 & 25.94 & 25.54 \\
& R.Medio & 197.12 & 196.50 & 189.31 & 196.25 & 197.10 \\
\hline & & \multicolumn{3}{c}{$\mathrm{Z}=2.83$} \\
\\
& & \multicolumn{5}{c}{$(\mathrm{p}=.005)$} \\
\hline Grupos & & Autoeficacia & Aprendizaje & Generatividad & P. total \\
\hline \multirow{2}{*}{ R. A. } & Media & 57.98 & 64.22 & 60.21 & 50.9 \\
(73) & D.S. & 29.73 & 17.09 & 26.01 & 26.0 \\
& R.Medio & 209.03 & 229.18 & 200.71 & 193.1 \\
R.G. & Media & 54.15 & 54.6 & 58.76 & 51.76 \\
(320) & D.S. & 29.06 & 28.56 & 28.98 & 25.47 \\
& R.Medio & 194.25 & 189.66 & 196.15 & 197.27 \\
\hline \multicolumn{5}{c}{$\mathrm{Z}=-2.69$} \\
\end{tabular}

En la escala de resiliencia se puede apreciar el puntaje obtenido por ambos grupos en las distintas subescalas. Solo en los factores modelos y aprendizaje se evidencian diferencias significativas. El estadístico obtenido de acuerdo a la prueba $\mathrm{U}$ de Mann-Whitney fue de $\mathrm{Z}=2.83(\mathrm{p}=.005)$ y $\mathrm{Z}=-2.69(\mathrm{p}=.007)$, respectivamente. En el factor modelo el grupo de estudiantes de rendimiento alto arroja un mejor puntaje. Esto se relaciona con la convicción del papel de las redes sociales cercanas para apoyar la superación de las situaciones problemáticas, personas con quienes se puede confiar, que ponen límites razonables a evitar peligros o problemas y brindar asesorías o consejos. El factor relacionado con aprendizaje se refiere a valorar la situación problemática como una posibilidad de aprendizaje y aprender de los aciertos y errores.

Tabla 4. Comparación del grupo de rendimiento alto (RA) con rendimiento general $(\mathrm{RG})$ en la escala de bienestar psicológico

\begin{tabular}{ccccccc}
\hline & & $\begin{array}{c}\text { Puntaje } \\
\text { total }\end{array}$ & Aceptación & Proyección & Vínculos & Control \\
\hline Rendimiento & Media & 34.18 & 7.40 & 8.47 & 7.95 & 10.38 \\
alto (RA) & D.S. & 2.84 & 1.35 & 1.00 & 1.09 & 1.53 \\
$(\mathrm{n}=73)$ & R. medio & 183.18 & 164.82 & 222.52 & 183.58 & 195.98 \\
Rendimiento & Media & 34.58 & 7.88 & 8.20 & 8.10 & 10.39 \\
general (RG) & D.S. & 2.85 & 1.11 & 1.04 & 1.05 & 1.55 \\
$(\mathrm{n}=320)$ & R. medio & 200.15 & 204.34 & 191.18 & 200.06 & 197.23 \\
\hline & & & $\mathrm{Z}=-2.80$ & $\mathrm{Z}=-2.35$ & & \\
& & & $(\mathrm{p}=.005)$ & $(\mathrm{p}=.019)$ & & \\
\hline
\end{tabular}

En la tabla anterior, se observan los puntajes obtenidos en la escala de bienestar psicológico. Los resultados indican que las diferencias se dan en las subescalas de aceptación y proyección. En la dimensión aceptación, el grupo de rendimiento 
alto obtiene una puntuación más baja que el grupo de rendimiento general. Los resultados se relacionan con la capacidad de aceptar los múltiples aspectos de sí mismos, incluyendo los buenos y malos, así como sentirse bien acerca de las consecuencias de los eventos pasados. Con respecto a la dimensión de proyección, se puede identificar que los estudiantes de rendimiento alto logran identificar la construcción de metas y proyectos, se evidencia un sentido y un valor por la vida, dotan de significado a las actividades que realizan y poseen noción y visión de futuro.

\section{DISCUSIÓN}

El estudio realizado confirma la relación entre la habilidad intelectual y el razonamiento con el rendimiento escolar. Aquellos alumnos que presentan un alto rendimiento académico dentro de su grupo curso poseen mejores habilidades para realizar comparaciones, razonar por analogías y organizar percepciones espaciales dentro de un todo relacionado.

Respecto a la autoestima se puede concluir que las diferencias solo se evidenciaron en el ámbito familiar. Se esperaba que existieran diferencias en el plano escolar y social, sin embargo, pareciera que el alto rendimiento de este grupo de estudiantes no se vincula con estos factores. En general, los que se destacan en los resultados académicos no participan activamente con sus compañeros; su foco es el estudio y, en general, son personas un poco más retraídas y aisladas (Morales y Morales Ma. 2013). La base teórica que sostenía esta hipótesis era los trabajos de Haeussler y Milicic (1995), al señalar que un buen nivel de autoestima genera alta motivación intrínseca, variable que facilita el aprendizaje, genera mayores expectativas sobre sí mismos y un mejor rendimiento académico. Sin embargo, los resultados no apoyaron esta hipótesis. El área escolar relacionada con la forma que el estudiante posee para afrontar las tareas académicas, como la capacidad para aprender y la satisfacción en sus tareas escolares tanto en el trabajo individual como grupal, da como resultado que los alumnos de rendimiento alto poseen un rendimiento más bajo que el grupo en general. Se sospecha que no basta poseer buenas calificaciones para alcanzar una autoestima alta en esta subescala, pues pareciera que el área social que se da en el contexto escolar es determinante.

El segundo factor estudiado fue la resiliencia. Son muchas las definiciones; sin embargo, Saavedra (2012) concluye que todas ellas reconocen el concepto como un fenómeno humano de carácter universal, que aparece frente a situaciones adversas que es necesario enfrentar y adaptarse, a pesar de los obstáculos, y proyectarse exitosamente. En el plano escolar la resiliencia se manifiesta en conductas de compromiso con el trabajo escolar, asistencia a clases, puntualidad, atención a la clase y al profesor, realización de las tareas escolares, altos niveles de autoconfianza, autodisciplina, actitud positiva hacia el trabajo, locus de control interno y autoestima positiva, rasgos que favorecen el aprendizaje.

Grotberg ( 2002) se refiere a las fuentes interactivas de la resiliencia, a las que clasifica en tres categorías: aquellas que tienen que ver con el apoyo que la persona puede recibir (yo tengo); aquellas que tienen relación con las fortalezas 
internas de la persona (yo soy) y aquellas que tienen que ver con las habilidades para relacionarse y resolver problemas (yo puedo). Estas fuentes interactivas se complementan con un modelo emergente desarrollado por Saavedra (2003), donde establece estas tres categorías anteriores junto con otros factores relacionados con condiciones de base, visión de sí mismo, visión del problema y respuesta resiliente.

Las diferencias entre los estudiantes de rendimiento alto y los estudiantes de rendimiento general se dan en el factor modelo (yo tengo). Los indicadores de este factor se relacionan con personas que lo han orientado, que lo han ayudado a evitar problemas, en quienes pueden confiar, demostraban altas expectativas y un constante acompañamiento al enfrentar diversos problemas.

El otro factor que arroja resultados significativos es en aprendizaje, factor que se relaciona con el yo puedo, aprender de sus aciertos y errores, colaborar con otros para mejorar la vida en la comunidad y tomar decisiones.

Respecto a la variable bienestar psicológico, las diferencias fueron en las dimensiones aceptación, relacionada con la capacidad para la toma de decisiones de modo independiente, la confianza que poseen los sujetos de su propio juicio, la estabilidad emocional, la capacidad para expresar lo que se piensa sin problemas y hacer lo que se quiere sin ser influenciable. Sin embargo, los puntajes fueron más altos en el grupo de rendimiento general que en el grupo de rendimiento alto.

En la dimensión proyectos, la diferencia entre ambos grupos también fue significativa. Se aprecia que los estudiantes de rendimiento alto poseen en la escala mayor puntuación que los estudiantes de rendimiento general en el establecimiento de metas, proyectos de vida. Consideran que la vida tiene valor y sentido.

Los resultados deben interpretarse en el contexto social, político y educacional que el país está experimentando. Los estudiantes han solicitado en su petitorio una nueva forma de acceso a la educación superior, con equidad, integración y heterogeneidad social y rechazan el actual sistema de selección universitaria (PSU) por su carácter regresivo y elitista. Aquellos alumnos que participaron en el estudio son jóvenes de 17 a 19 años, de cuarto año de enseñanza media (corresponde al último año de la enseñanza media) y pertenecen al I y II quintil del nivel per cápita familiar. Son estudiantes que están más expuestos a estados de frustración, ansiedad e inseguridad debido al contexto social y selectivo de la educación superior. A pesar del deseo de insertarse a la universidad o iniciar estudios en educación superior, su acceso les es difícil, producto de múltiples factores que contribuyen a que su proyecto de vida se transforme en algo inestable, impredecible e inseguro, lo que interfiere en su bienestar psicológico.

A partir de este estudio, son muchas las nuevas interrogantes que surgen. Futuras investigaciones permitirían conocer los sucesos vitales normativos y no normativos que han tenido que enfrentar y cómo estos han sido vividos, lo que influirían en diversas dimensiones del bienestar: dominio del medio, relaciones positivas, propósitos en la vida, autoaceptación, crecimiento personal, autonomía, entre otras. El valor de estos estudios contribuirían a instaurar competencias que los jóvenes 
adolescentes pudiesen incorporar a su vida para enfrentar situaciones diversas y adversas que deben vivir durante las distintas etapas de su desarrollo, especialmente en esto sectores de alto grado de vulnerabilidad.

\section{CONCLUSIONES}

El estudio realizado confirma la relación de la habilidad intelectual y el razonamiento con el rendimiento escolar.

Respecto a la autoestima se puede concluir que las diferencias solo se evidenciaron en el ámbito familiar. El subtest autoestima familia-hogar aparece favorable para los estudiantes de rendimiento alto. El resultado indica que este grupo de alumnos con rendimiento alto demuestra adecuadas cualidades y habilidades en las relaciones íntimas con la familia; el sujeto se siente respetado, tiene independencia y una concepción moral propia. Este respeto podría ser consecuencia del rendimiento escolar que el individuo ha alcanzado, lo que permite una alta valoración en esta área sobre sí mismo.

Con relación a la variable resiliencia, las diferencias entre los estudiantes de rendimiento alto y los estudiantes de rendimiento general se dan en el factor modelo y aprendizaje. El primero de ellos se relaciona con la visión que el individuo tiene del problema y el entorno. Se trata de estudiantes que reconocen personas que los han orientado, aconsejado y acompañado, que los han ayudado a evitar peligros y en quienes pueden confiar. El factor aprendizaje se relaciona con aprender de los aciertos y errores, tomar decisiones y mejorar los procesos de comunicación.

En bienestar psicológico las diferencias se encontraron en la dimensión aceptación. El grupo de rendimiento general presenta puntajes más altos que los estudiantes con rendimiento alto. En la dimensión proyectos, la diferencia entre ambos grupos también fue significativa. Se aprecia que los estudiantes de rendimiento alto poseen en la escala mayor puntuación que los estudiantes de rendimiento general.

Nota de reconocimiento: El autor agradece a la Dirección de Investigación y Tecnología (Dicyt) de la Universidad de Santiago de Chile, por su apoyo en la aprobación y desarrollo de la investigación.

\section{REFERENCIAS BIBLIOGRÁFICAS}

Acosta, R. y Hernández, J. (2000). La autoestima en la educación. Revista Límite, vol. 1, número 001, Universidad de Tarapacá, Arica, Chile, pp. 82-95.

Aliaga, J. (1998). La inteligencia, la personalidad y la actitud hacia las matemáticas y el rendimiento en matemáticas de los estudiantes del quinto año de secundaria. Un enfoque multivariado. Tesis de Maestría en Educación. Universidad de San Martin de Porres, Lima.

Barber, B. L. \& Weichold, K. (2006). Introduction to research on interventions targeting the promotion of positive development. Research on interventions targeting the 
ESTUDIANTES DE SECTORES VULNERABLES CON ALTO RENDIMIENTO ESCOLAR Y SU DESEMPEÑO EN VARIABLES INTELECTUALES...

promotion of positive development. Internacional Society for the Study of Behavioural Development Newsletter, 50(2), 1-4.

Brinkmann, H., T. Segure y M. I. Solar (1989). Adaptación y estandarización del Inventario de Autoestima de Coopersmith, Revista Chilena de Psicología 10 (1): 73-87.

Casullo, M. (2000). Evaluación del Bienestar Psicológico en Iberoamérica. Buenos Aires, Argentina: Paidós.

Dew, T. y Scott, E. (2002). La calidad de vida percibida en Adolescentes: una investigación exploratoria. Universidad de Carolina del Sur.

Diener, E. \& Lucas, R. (2000). Personality and subjetive well-being. Russel Sage Foundation: New York.

Diener, E. (1994). Assessing Subjective Well-Being: Progress and Opportunities. Social Indicators Research, 31, 103-157.

Diener, E., Suh. E., Lucas, R. \& Smith, H. (1999). Subjective Well-Being: Three Decades of Progress. Psychological Bulletin, 125 (2), 276-302.

Elder, G. H., Van Nguyen, T., \& Caspi, A. (1985). Linking family hardship to children's lives. Child Development, 56(2), 361-375.

Finn, J. D. y Rock, D. A. (1997). Academic success among students at risk for school failure. Journal of Applied Psychology, 82, 221 - 234.

Fredrickson, B. L. \& Joiner, T. (2002). Positive emotions trigger upward spirals toward emotional well-being. Psychological Science, 13, 172-175.

García, M. (2004). Desde el concepto de felicidad al abordaje de las variables implicadas en el bienestar subjetivo: un análisis conceptual. [Disponible en Red]. www.efdeportes.com/.

Grotberg, E. (2002). Nuevas tendencias en resiliencia. En Melillo, A y Suárez, E., (comp), Resiliencia: Descubriendo las propias fortalezas. (pp. 19-30). Buenos Aires: Paidós

González-Pienda, J. (2003) "El rendimiento escolar. Un análisis de las variables que lo condicionan”. Revista Galego-Portuguesa de Psicologia Educación N 7(Vol8) 247-258

Grotberg, E. (1996). A Guide to Promoting Resilience in Children: strengthening the spirit human. La Haya: Bernard Van Leer Foundation.

Groetberg, E. (2006) ¿Qué entedemos por Resiliencia? ¿cómo promoverla? ¿cómo utilizarla? Barcelona:Gedisa

Haeussler, I. y Milicic N. (1995). Confiar en uno mismo. Programa de desarrollo de la autoestima. Santiago: Editorial Dolmen.

Kotliarenco, M., Cáceres, I. y Fontecilla, M. (1996). Estado de arte en Resiliencia [versión electrónica]. Organización Panamericana de la Salud. Washington D.C.: OPS/OMS, Fundación W. K. Kellogg, CEANIM.

Masten A.S.(2001) Ordinary magic. Resilience processes in development. American Psychologist, 56, 227-239 
Masten, A. (1994). Resilience in individual development: Successful adaptation despite risk and adversity. En M. C. Wang \& E. W. Gordon (Eds.), Educational resilience in inner-city America: Challenges and prospects (pp. 3-26). Hillsdale, NJ: Erlbaum.

Morales, M., Morales, Ma. (2013) Adolescentes de buen rendimiento escolar de liceos vulnerables y la resignificación de su experiencia escolar. En $2^{\circ}$ Congreso Alfepsi. Construyendo una Psicología comprometida con América Latina. Concepción. Chile. 1903-1938

Naranjo, M. (2007). Autoestima: Un factor relevante en la vida de la persona y tema esencial del proceso educativo. Revista Electrónica Actualidades Investigativas en Educación, septiembre-diciembre, año/vol. 7, número 003. Universidad de Costa Rica. San José, Costa Rica.

Polleti, R. y Dobbs, B. (2005). La autoestima. Un bien esencial. Buenos Aires: Lumen.

Raven, J. (1991). Test de matrices progresivas para la medida de la capacidad intelectual (de sujetos de 12 a 65 años), Manual. Buenos Aires: Paidós

Rodríguez, C. y Valdivieso, A. (2008). El éxito escolar de alumnos en condiciones adversas. Revista Latinoamericana de Estudios Educativos (México), XXXVIII, 81-106.

Rutter, M. (1999). Resilience concepts and findings: Implications for family therapy. Journal of Family Therapy, 21, 119-144.

Saavedra, E. (2003). La Emoción como Construcción de Significados. Patio, Revista Pedagógica. $\mathrm{N}^{\circ} 27$ Agosto- octubre. Brasil.

Saavedra, E. (2012).Una aproximación al estado del arte de la investigación en resiliencia. En J. Catalán. (Ed.). Investigación orientada al cambio en Psicología Educacional. La Serena: Universidad de la Serena.

Sameroff, A. J., Bartko, W. T., Baldwin, A., Baldwin, C. \& Seifer, R. (1998). Family and social influences on the development of child competence. En M. Lewis y C. Feiring. (Eds.), Families, risk and competence (pp. 161-185). Mahwah, NJ: Lawrence Erlbaum.

Seligman, M. (2005). La auténtica felicidad. Buenos Aires: Ediciones Byblos

Téllez, E. (2005) "Desigualdad social y pobreza de alumnos de escuela primaria", Tesis para optar al grado de doctora en Ciencias de la Educación, Pachuca, Hidalgo, Universidad Autónoma del Estado de Hidalgo, México.

Torre de Carvalho, F., Araujo de Morais, N., Koller, S. H. y Piccinini, C. A. (2007). Fatores de proteçao relacionados à promoção de resiliência em pessoas que vivem com HIV/Aids. Cadernos de Saúde Pública 23(9), 2023-2033.

Villalta, M. (2009) Una propuesta para el estudio de la interacción didáctica en la sala de clase. Estudios Pedagógicos. Vol. XXXV(1), 221-238.

Werner, E. (1990). Protective factors and individual resilience. En S. Meisels \& J. Shonkoff (Eds.), Handbook of early childhood intervention (pp. 115-133). New York: Cambridge University Press. 